\title{
The preventive effect of sensorimotor- and vibration exercises on the onset of Oxaliplatin- or vinca-alkaloid induced peripheral neuropathies - STOP
}

Fiona Streckmann ${ }^{1,2,3^{*}}$ (D, Maryam Balke ${ }^{4}$, Helmar C. Lehmann ${ }^{4}$, Vanessa Rustler ${ }^{1}$, Christina Koliamitra', Thomas Elter ${ }^{5}$, Michael Hallek ${ }^{5}$, Michael Leitzmann ${ }^{6}$, Tilman Steinmetz ${ }^{7}$, Petra Heinen ${ }^{8}$, Freerk T. Baumann ${ }^{5}$ and Wilhelm Bloch ${ }^{1}$

\begin{abstract}
Background: Chemotherapy-induced peripheral neuropathy (CIPN) is a common and clinically relevant side effect of chemotherapy. Approximately 50\% of all leukemia, lymphoma, colorectal- and breast cancer patients are affected. CIPN is induced by neurotoxic chemotherapeutic agents and can manifest with sensory and/or motor deficits. It is associated with significant disability and poor recovery. Common symptoms include pain, altered sensation, reduced or absent reflexes, muscle weakness, reduced balance control and insecure gait.

These symptoms not only affect activities of daily living, subsequently reducing patients' quality of life, they have far more become a decisive limiting factor for medical therapy, causing treatment delays, dose reductions, or even discontinuation of therapy, which can affect the outcome and compromise survival. To date, CIPN cannot be prevented and its occurrence presents a diagnostic dilemma since approved and effective treatment options are lacking. Promising results have recently been achieved with exercise. We have revealed that sensorimotor training (SMT) or whole body vibration (WBV) can reduce the symptoms of CIPN and attenuate motor and sensory deficits. We furthermore detected a tendency that it may also have a preventive effect on the onset of CIPN.

Methods: We are therefore conducting a prospective, multicentre, controlled clinical trial involving 236 oncological patients receiving either oxaliplatin $(N=118)$ or vinca-alkaloid $(N=118)$ who are randomized to one of two interventions (SMT or WBV) or a treatment as usual (TAU) group. Primary endpoint is the time to incidence of neurologically confirmed CIPN. Secondary endpoints are pain, maintenance of the functionality of sensory as well as motor nerve fibres as well as the level of physical activity. The baseline assessment is performed prior to the first cycle of chemotherapy. Subsequent follow-up assessments are conducted at 12 weeks, after completion of chemotherapy, and at a 3-month follow-up. Patients who develop CIPN receive an additional assessment at this time point, as it represents the primary endpoint.
\end{abstract}

Discussion: We hypothesize that SMT and WBV prevent the onset or delay the progression of CIPN, decrease the likelihood of dose reductions or discontinuation of cancer treatment and improve patients' quality of life.

Trial registration: Deutsche Register Klinischer Studien (DRKS00006088, registered 07.05.2014).

Keywords: Exercise, Neuromuscular, Sensory deficits, Motor performance, Quality of life, Cancer therapy, Neurotoxic agents, Physical activity

\footnotetext{
*Correspondence: f.streckmann@dshs-koeln.de; fiona.streckmann@unibas.ch; fiona.streckmann@usb.ch

'Institute for Cardiovascular Research and Sports Medicine, German Sport

University Cologne, Am Sportpark Müngersdorf 6, 50933 Cologne, Germany

${ }^{2}$ Department of Sport, Exercise and Health, University of Basel, Birsstr. 320B,

4052 Basel, Switzerland

Full list of author information is available at the end of the article
} 


\section{Background}

Chemotherapy-induced peripheral neuropathy (CIPN) is caused by neurotoxic agents in cancer therapy. Oxaliplatin and vinca-alkaloids are two of the main agents responsible for CIPN. Oxaliplatin inhibits DNA synthesis and repair due to its ring structure, which causes the death of neural cells. Vinca-alkaloids cause axonal damage and disrupt axonal transport via microtubular damage. The main cancer patients affected by oxaliplatin are colorectal, NHL and breast cancer patients, while lymphoma patients but also ALL and pulmonary cancer patients mainly receive vinca-alkaloids. Peripheral sensory nerves are especially sensitive to toxins. Damage caused to these fibres leads to various sensory and motor dysfunctions. Patients suffer from symptoms such as loss of sensation, apparent as numbness, tingling or burning, dysaesthesia, reduced or absent Achilles tendon reflexes [1,2] pain, and loss of balance control leading to instable gait, as well as an increased incidence of accidents and falls [3].

Even though CIPN is such a prevalent and clinically relevant side effect [4], not only diminishing patients' quality of life, but also leading to treatment delays, dose reductions or even discontinuation of therapy, affecting the outcome and compromise survival [5], little research has been done to investigate the potentially beneficial effects of specific exercises to counteract the various motor and sensory dysfunctions.

To date, CIPN cannot be prevented and there is no consent regarding the treatment of CIPN. Research has focused on pharmacological therapies aimed at reducing CIPN or treating selected side effects while [6-8] this has been helpful for neuropathic pain, it does not address the many other side effects of CIPN [9-12]. On the contrary, many of these agents have been shown to have additional negative side effects [13]. An exercise intervention has now revealed promising results. In a first clinical trial, we [14] conducted an exercise intervention consisting of endurance, strength and sensorimotor training (SMT) twice a week for 36 weeks, accompanying lymphoma patients from diagnosis to completion of treatment. The study revealed a significant reduction of neuropathic symptoms. Patients exercising were able to reduce CIPNrelated symptoms (e.g., peripheral deep sensitivity) by $87 \%$, while in the control group no change $(0 \%)$ was detected. After 36 weeks, 55\% of the control group still had symptoms related to CIPN while only $4 \%$ remained with CIPN in the intervention group.

Furthermore, a positive tendency regarding the incidence of CIPN could be detected. Unfortunately, the sample size was too small in this study to show significant results. The majority of expertise on exercise and peripheral neuropathy (PNP) arises from research on patients with diabetic neuropathy. In a systematic review [15], we evaluated all exercise intervention studies for neuropathic patients independent of the cause. We found that for toxically induced PNP such as CIPN, balance exercises were most beneficial for motor as well as sensory symptoms.

Taking previous findings into consideration, this strengthened our presumption that SMT played a decisive role in the study by Streckmann et al. [14], as studies in healthy adults have revealed that SMT has the potential to counteract some of the mentioned side-effects of PNP. SMT is characterized by functional adaptations of the neuromuscular system $[16,17]$, regeneration of neuromuscular structures [18] and the diminished prevalence of injuries $[19,20]$, leading to improved proprioception [17], intermuscular coordination and balance control, causing fewer falls [21] and increasing mobility. Furthermore, studies with strength training alone or in combination with endurance training showed little to no significant intergroup differences. In line with these findings, Steimann [22] and Vogt [4] evaluated the subjective effectiveness of physiotherapy (gait training and balance exercises) and ergotherapy (e.g., walking on granulate material), while Steimann also looked at electrotherapy. Both found that patients experienced ergotherapy and physiotherapy as very helpful. One case report on a breast cancer patient, suffering from painful CIPN, showed improved balance after balance training [23].

Targeting similar mechanisms as SMT, though possibly addressing different sensory qualities, whole body vibration (WBV) has also been taken into consideration. Previous studies investigating WBV have shown a positive impact on parameters influenced by the side-effects of PNP. Kawanabe et al. [24] and Bogaerts et al. [25] showed that elderly individuals improve their gait after vibration exercises. Rittweger [26] and Kirchner et al. [27] found WBV to have a positive impact on pain reduction, while further studies showed an effect on deconditioned skeletal muscle [28], improved isometric strength [26, 29, 30], postural sway [31] and reduced fall frequency [25]. Schönsteiner et al. [32], performed a multimodal exercise program containing WBV, massage and physical exercises with CIPN patients $(N=131)$, achieving less symptoms and pain, improved physical fitness and better coordination. Both SMT and WBV require very little time and effort, but have a high impact. Especially for cancer patients, this aspect plays an important role, as therapy can be very strenuous for the patients. Training and devices are feasible, meet the requirements of hospital hygiene and are portable for all phases of therapy, even in isolation. Training is therefore even possible during cytopenias, often a limiting factor for exercise interventions concomitant to therapy.

We therefore conducted a randomized, controlled, pilot study assessing cancer patients with neurologically confirmed CIPN to either SMT $(n=10), \mathrm{WBV}(n=10)$ 
or a control group $(\mathrm{n}=10)$ with no intervention additionally comparing them to an age- and gender matched healthy control group $(n=10)$. WBV and SMT were feasible for patients with CIPN and both exercise groups benefited (improved reflex activity of the Achilles- and patella tendon), peripheral deep sensitivity and pain) from 6 weeks of intervention twice a week [33].

To summarize, there are no existing prevention trials assessing the potentially beneficial effects of exercise for the onset of CIPN and only very little is known about the effects of exercise on the symptoms of CIPN. Based on our previous findings as well as from practical experience with patients, we hypothesize that SMT and WBV prevent the onset of CIPN on the one hand and/or can influence the progress of CIPN and associated motor and sensory symptoms such as balance control, coordination and mobility, as well as sensitivity, proprioception and pain, enhancing patients' quality of life and assuring the best clinical outcome by enabling patients to receive their planned therapy regimen.

\section{Methods/design}

\section{Study participants and recruitment}

We plan to enrol 236 newly diagnosed haematological/ oncological patients who are scheduled to receive chemotherapy containing either oxaliplatin or a vincaalkaloid, aged $\geq 18$ years, with the mental and physical ability to provide signed informed consent and participate in the study. Patients are recruited at three participating centres: The University Hospital of Cologne, the St. Antonius Hospital in Eschweiler and the joint practice for oncology and hematology at the Sachsenring in Cologne. Exclusion criteria is a pre-existing neuropathy of other cause. Therefore, patients will be assessed clinically for signs of neuropathy and will undergo nerve conduction studies prior to randomization. Neuropathy will be defined electrophysiologically as CMAP amplitude below $5 \mathrm{mV}$, SNAP amplitude below $5 \mu \mathrm{V}$, and nerve conduction velocity below $40 \mathrm{~m} / \mathrm{s}$ of tibial or sural nerve). Further exclusion criteria are previous therapies containing neurotoxic agents, any contraindication for whole body vibration (instable bone metastases, acute leg thrombosis, a fracture in the lower extremities in the past 2 years, foot ulcers, artificial hips or other osteosynthesis), and myocardial infarction, angina pectoris or heart disease (NYHA III-IV) within the past six months.

\section{Experimental design}

The study follows a prospective, randomized controlled design, allocating patients to three groups: an intervention group receiving SMT, an additional intervention group receiving WBV, and a control group (Fig. 1). Patients in the two intervention groups receive a defined exercise program twice a week in addition to treatment as usual (TAU). Patients in the control group receive TAU and are given the opportunity to participate in the preferred intervention after completion of the study. The interventions and assessments take place at the respective centers.

Data is assessed at 3 to 5 measuring time points, depending on the length of medical therapy and a potential incidence of CIPN (Fig. 2 and Fig. 3). The baseline assessment (T0) is performed prior to the first cycle of chemotherapy. All patients are re-assessed after three months (T1). For most patients, this is simultaneously the post-therapy measurement (Tp) (Fig.2), while for patients who are treated for more than three months it represents an interim assessment (T1) (Fig.3), in order to ensure comparability regardless of the entity. These patients have an additional assessment upon completion of their medical therapy ( $\sim 6$ months). The follow-up measurement is performed three months after completion of chemotherapy (T2) in order to compensate for any potential coasting effects. Each assessment has a duration of $90 \mathrm{~min}$ at most. To ensure the detection of CIPN, patients are informed about possible symptoms of CIPN and asked to report back to the study coordinators immediately. Furthermore, patients are regularly asked for potential symptoms by their physicians. Additionally, a short neurological test battery is performed every 6 weeks. Sports therapists will be blinded and must not ask patients about CIPN symptoms during the interventions in order to obtain comparability with the control group. In case a CIPN is neurologically confirmed, patients are also tested at this time point (Ti).

\section{Assessment - primary endpoint}

In order to assess the time to incidence of a neurologically confirmed CIPN, a comprehensive Neurophysiological assessment that includes the entire symptom pattern of CIPN, is necessary (Table 1):

Nerve conduction studies are performed by trained, certified and blinded examiners. For patients of the University Hospital Cologne as well as the joint practice at Sachsenring, neurophysiological assessments are performed in the Electrophysiology Laboratory of the Department of Neurology, University Hospital Cologne. Patients in Eschweiler are seen by a local neurologist. Assessment methods are standardized and aligned among the investigators. Furthermore, patients are asked not to mention the arm they are participating in to the investigators. Examiners are trained by a gold-standard examiner using a standard operating procedure and certified prior to the study.

\section{Nerve conduction studies}

For nerve conduction studies, motor and sensory nerves are assessed. Compound muscle action potentials (CMAP), distal motor latency, conduction velocity, and F-waves are obtained from the tibial nerve. 
The tibial nerve is stimulated at the ankle and popliteal fosse. Antidromic sensory nerve conduction studies are performed in the sural nerve. Sensory nerve action potentials (SNAPs) are recorded from the lateral malleolus with surface electrodes. Skin temperature is monitored and maintained above $32{ }^{\circ} \mathrm{C}$ using a heater if necessary.

We furthermore conduct a standardized neurological clinical test battery that is a feasible assessment method for oncological patients in order to check for first neuropathic symptoms. It is used as a pretest to screen for CIPN related symptoms. Should one of the components show irregularities, a neuroelectrography is required in order to detect and document a possible CIPN.

The test battery contains the following assessments:

1. Peripheral deep sensitivity is evaluated by the use of a Rydel-Seiffer tuning fork $(128 \mathrm{~Hz})$ with a scale from 0 to 8 . Due to age related neural deconditioning, values $\leq 4$ are pathological for patients $\geq 60$ years old, while for patients under 60 years old, $\leq 5$ is regarded as pathological [34].

2. The Reflex action of the Achilles- as well as the patellar tendon is assessed with a reflex hammer

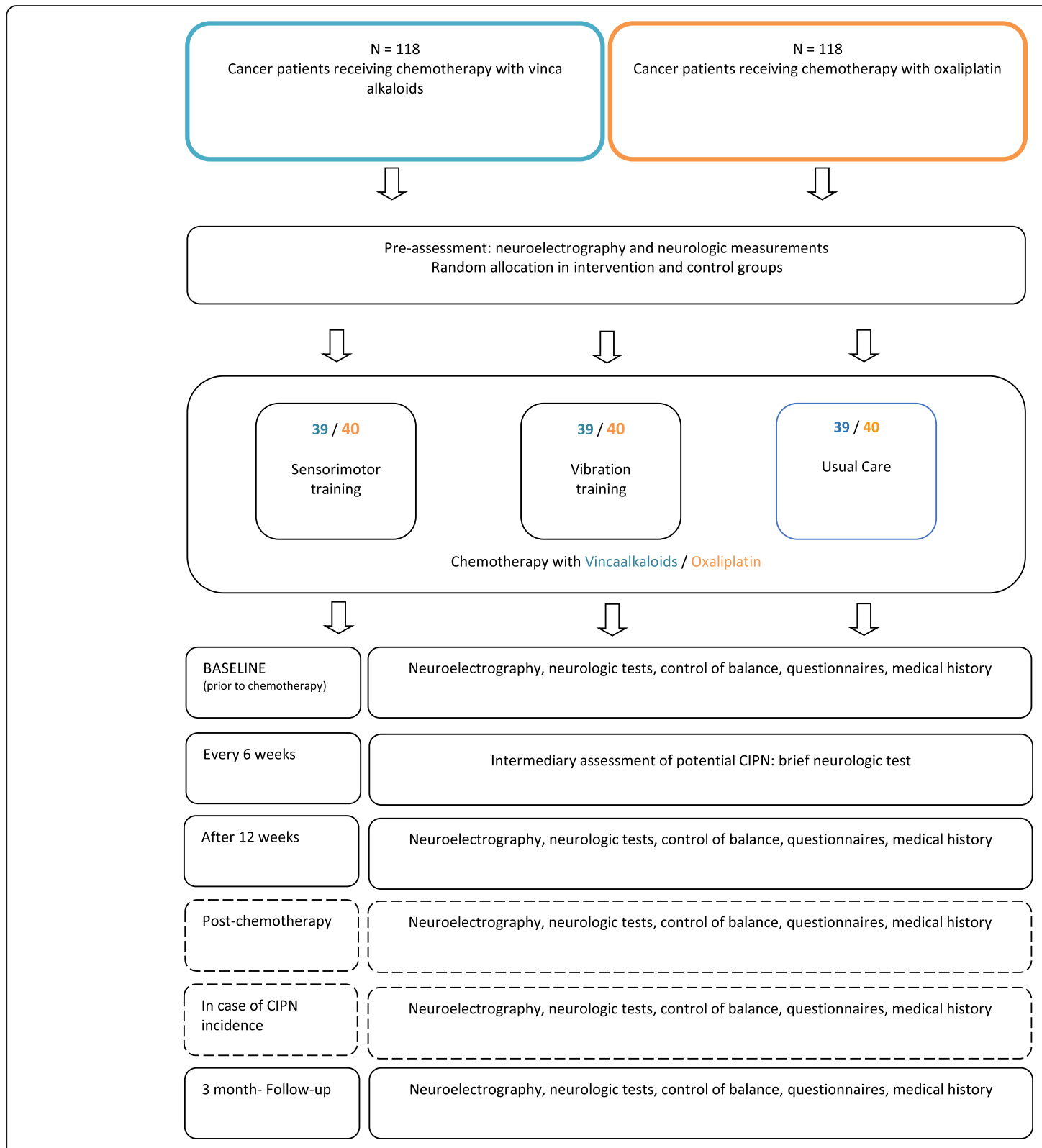

Fig. 1 Overview of the study design 


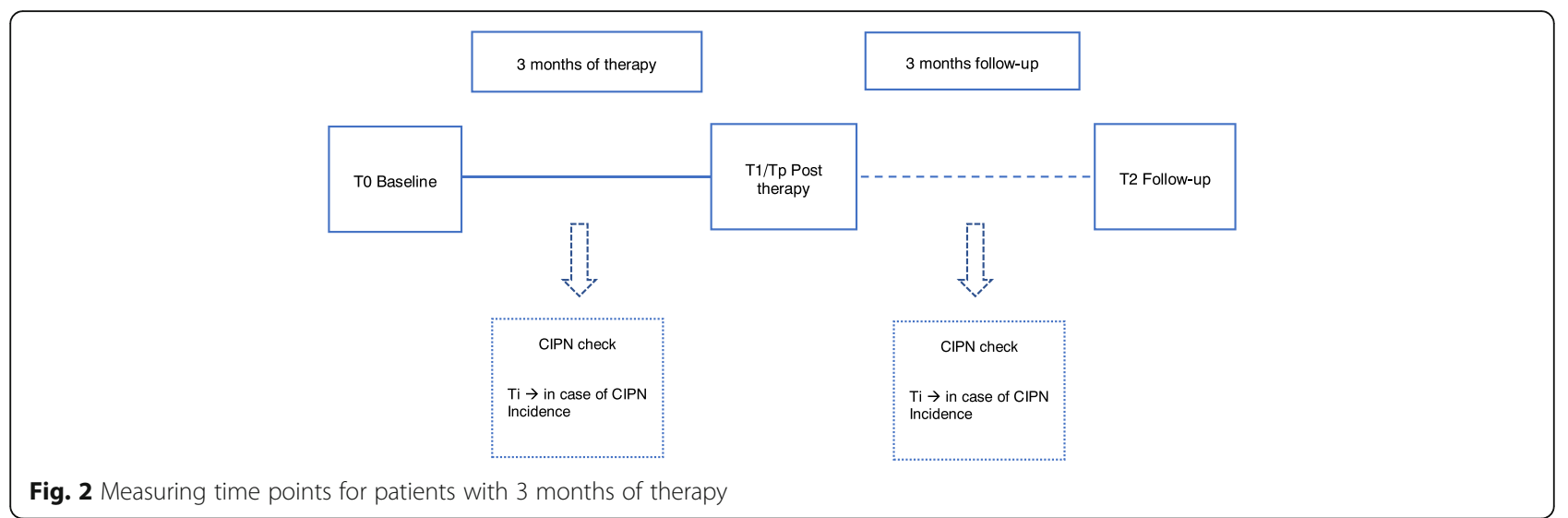

and graded on a 3 -point scale $(1=$ agile, $2=$ weak, 3 $=$ missing).

3. Sense of position is assessed by asking patients if they can recognize a change of position in their first toe, with their eyes closed.

4. Perception of touch is evaluated by symmetrically stroking the outsides of the patients' legs and feet in order to detect reduced or altered sensation due to demyelination or axonal degeneration [30, 35].

\section{The strength of the lower leg muscles is} assessed by requesting the patient to actively move their legs against the resistance of the examiner's arm. The examiner then grades the strength on a six-point scale $(0=$ no activity, $1=$ visual contraction without motor effect, $2=$ movement under elimination of gravity, $3=$ movement under gravity, $4=$ movement against slight resistance $5=$ normal force).

\section{Assessment - Secondary endpoints}

\section{Postural control}

A force plate (Leonardo Mechanograph ${ }^{\circ}$, Novotec medical, Pforzheim, Germany) is used to assess changes in the center of pressure during upright static and dynamic stance. The assessment follows a standardized protocol (see Table 1). Primarily, the supporting foot is determined with a short test [36]. Patients are asked to maintain an upright position with their knees slightly flexed $\left(\sim 30^{\circ}\right)$, hands at their side and their gaze straight ahead for $30 \mathrm{~s}$. The cumulative change in sway paths during this period is registered and serves as a measure of postural control. To minimize bias through potential learning effects, each position is repeated three times. Additionally, failed attempts are recorded should a patient seek hold. The tasks become progressively more difficult as previous studies (see reference [37] for review) have shown that postural tasks with different complexity serve best to test for changes in stance stability after balance training. To assess the dynamic stance, a balance pad is additionally placed on top of the force plate.

\section{Questionnaires}

FACT/GOG-Ntx - questionnaire The particular sector of the FACT/GOG-Ntx [Functional Assessment of Cancer Therapy/Gynaecology Oncology Group Neurotoxity] is used to document and assess the severity of the subjective PNP symptoms [38]. This questionnaire has been validated and contains eleven items which allow an assessment of the extent of PNP symptoms from "not at all" to "very much" [25].

EORTC-QLQ-CIPN20 The EORTC-QLQ-CIPN20 is a phase IV questionnaire that we are evaluating for $\mathrm{N}$. Aaronson in the course of this study. It is a 20-item questionnaire that was developed to elicit patients'

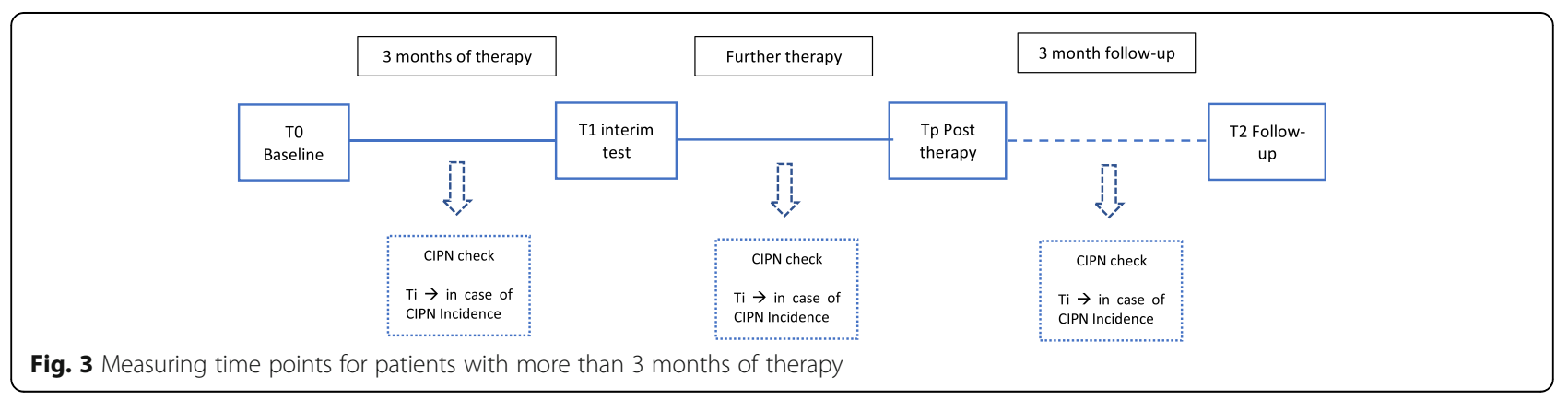


Table 1 Flow-chart of assessments

\begin{tabular}{|c|c|c|c|c|c|c|c|}
\hline & & Baseline T0 & T1 & $T p$ & T2 & $\mathrm{Ti}$ & $\begin{array}{l}\text { Status } \\
\text { measurement }\end{array}$ \\
\hline Time points & & $\begin{array}{l}\text { Prior to first } \\
\text { cycle of } \\
\text { therapy }\end{array}$ & After 3 months & $\begin{array}{l}\text { After medical } \\
\text { therapy }\end{array}$ & $\begin{array}{l}3 \text { month } \\
\text { follow-up }\end{array}$ & $\begin{array}{l}\text { Incidence } \\
\text { CIPN }\end{array}$ & every 6 weeks \\
\hline Intervention & Training & \multicolumn{3}{|c|}{$2 \times$ per week throughout entire medical therapy } & $\begin{array}{l}\text { Patients } \\
\text { offered to } \\
\text { continue }\end{array}$ & & \\
\hline Anamnesis I & $\begin{array}{l}\text { Entity, stadium, pre-treatment, } \\
\text { pre-diseases, allergies, planed } \\
\text { therapy, neurological anamnesis, } \\
\text { CIPN relevant medication, social } \\
\text { anamnesis }\end{array}$ & \multicolumn{3}{|l|}{$x$} & & & \\
\hline Anamnesis II & $\begin{array}{l}\text { Begin of CIPN Symptoms, } \\
\text { reception of planed therapy. } \\
\text { Amount of cycles, potential } \\
\text { change of medication or therapy, } \\
\text { CIPN relevant medication }\end{array}$ & & & $x$ & $x$ & $x$ & \\
\hline Anamnesis III & $\begin{array}{l}\text { Reception of CIPN relevant } \\
\text { medication, therapy of CIPN }\end{array}$ & & & Only CG & Only CG & Only CG & \\
\hline \multirow[t]{2}{*}{$\begin{array}{l}\text { Neurological } \\
\text { assessment }\end{array}$} & $\begin{array}{l}\text { Neuroelectrography } \\
\text { (NCV, Amp) }\end{array}$ & $x$ & $x$ & $x$ & $x$ & $x$ & \\
\hline & $\begin{array}{l}\text { Neurological clinical tests } \\
\text { battery }\end{array}$ & $x$ & $x$ & $x$ & $x$ & $x$ & $x$ \\
\hline $\begin{array}{l}\text { Performance } \\
\text { status }\end{array}$ & $\begin{array}{l}\text { Static and dynamic postural } \\
\text { control }\end{array}$ & $x$ & $x$ & $x$ & $x$ & $x$ & \\
\hline Questionnaires & $\begin{array}{l}\text { Subjective reduction of } \\
\text { symptoms (FACT/GOG-Ntx / } \\
\text { EORTC CIPN 20) } \\
\text { Quality of life (EORTC QLQ-C 30) } \\
\text { Neuropathic pain (PainDETECT } \\
\text { and VAS) } \\
\text { Level of physical activity (FFKA) }\end{array}$ & $x$ & $x$ & $x$ & $x$ & $x$ & \\
\hline
\end{tabular}

CIPN Chemotherapy-induced peripheral neuropathy, CG control group, NCV nerve conduction velocity, Amp amplitude, VAS visual analogue scale

experience of symptoms and functional limitations related to CIPN.The CIPN20 has 3 subscales: a sensory, a motor, and an autonomic subscale.

EORCT-QLQ-C30 The EORTC-QLQ-C30-questionnaire is used to assess health-related quality of life. In addition to a scale for "global quality of life", the questionnaire contains five functional scales (physical, emotional, social and cognitive functions, and role functions), three symptoms scales (fatigue, pain, nausea/ vomiting), and single item scales of respiratory distress, insomnia, loss of appetite, constipation, diarrhoea, and financial problems. The questionnaire has been validated and translated into 81 languages and has been used in more than 3000 studies worldwide. It is internationally regarded as reliable $[39,40]$.

PainDETECT This questionnaire focuses on pain specifically related to PNP. It helps assess patients' subjective experience of neuropathy-related pain. The questionnaire includes 12 items that take the intensity, progression, and distribution of pain into account. The questions are answered on a Likert scale ranging from "not at all" to "very much", which are summed up to yield a total score that reflects neuropathic pain status. Pain DETECT is a validated and reliable screening tool with high sensitivity, specificity and positive predictive accuracy $[41,42]$.

FFKA Physical activity levels are evaluated using the Freiburger Physical Activity Questionnaire (FFKA), a standardized and validated questionnaire that assesses the physical activities performed by a patient during the past 4 weeks. Based on the patients' answers, METscores are calculated $[43,44]$.

See Table 1 for Flow-chart of all assessments.

\section{Training program}

The interventions start immediately after randomization and are continued throughout the entire medical therapy ( $\sim 3$ to 6 months). Training sessions are supervised and take place twice a week in specific training rooms designed to meet the needs of oncological patients in an outpatient setting or during the hospital stay, in one of the 
centers. Each session lasts for about 15 to $30 \mathrm{~min}$. Depending on the type of intervention, the training will involve:

Sensorimotor training consists of progressively more difficult balance exercises on progressively instable surfaces. Each patient performs 4 exercises per session following a standardized protocol (see Table 1). Each exercise is performed three times for $20 \mathrm{~s}$, allowing a $40 \mathrm{~s}$ rest between each set and a 3-min rest between each exercise, to avoid neuronal fatigue. Patients are asked to stand barefoot or in socks, their foot in a previously acquired "short-foot-position", knees slightly flexed $\left(30^{\circ}\right)$, and to maintain balance.

Vibration training takes place on a vibration platform $\left(\text { Galileo }^{\mathrm{TM}} \text {, Pforzheim, Germany }\right)^{\bullet}$. Each training session consists of four sets of $30 \mathrm{~s}$ to $1 \mathrm{~min}$ vibration. The frequency of the vibrating platform ranges between 18 and $35 \mathrm{~Hz}$ with a $2 \mathrm{~mm}$ amplitude. Between sets, the patients rest for at least $1 \mathrm{~min}$ to avoid fatigue. Patients are asked to stand on the platform barefoot and on their forefeet or if they are too instable, an $80 / 20 \%$ distribution of weight on the forefeet rather than the heels.

Each training session allows for individual progression within a standardised selection of exercises (see Table 1) and is documented by the sports therapist.

\section{Statistical procedures and sample size estimation}

Central computerized randomisation (RITA) using a modified minimization procedure with stochastic component according to Pocock and Simon is performed [45]: intervention 1: intervention 2 :control $=1: 1: 1$, stratified by study center and type of therapy (Oxaliplatin, Vinca-alkaloids). In trials under similar conditions, a balanced randomization has been achieved using this algorithm [14].

Sample size calculation is based on the primary endpoint incident CIPN. Power calculation is based on the following scenario: The assumed incidence rate with TAU is $90 \%$, which was informed by a review of the literature. In both intervention groups, we assumed an incidence rate of $75 \%$. The effect size corresponds to a relative risk of about 0.60 , which is a clinically meaningful effect size. Using the log-rank test $(1-\beta=$ 0.8 , two-sided $\alpha=0.05$ ), we need a total of 196 evaluable patients, 65 per group $[46,47]$. We anticipate a drop-out rate of $10 \%$, yielding a total of 236 patients to be recruited for this study, 79 per group. This calculation is conservative as we may achieve additional power performing the final analysis using a multivariable Cox proportional hazards regression model adjusting for study center, type of chemotherapy, type of cancer, gender, and age.

\section{Recruitment of patients}

Patients are recruited in three centers: The University Hospital Cologne/ CIO Cologne Bonn, the St.-AntoniusHospital in Eschweiler, and the Oncological Practice at the Sachsenring in Cologne. The numbers of patients are based on the average number of patients in the respective centers per year over the past 2 years, considering denial or drop-out and applied to the recruitment period.

\section{Data management and analyses}

Data entry is continuously monitored by a data manager (TN) and will be analyzed by a statistician (ML). For the primary endpoint incident CIPN, censoring will be taken into account by using log-rank tests, and multivariable Cox proportional hazard regression models will be used to test for differences between groups and to estimate treatment effects. For categorical secondary endpoints, Fisher's exact test and Wilcoxon signed-rank tests and multivariable logistic regression models will be performed. For continuous outcomes (including scores derived from selfreport questionnaires), t-tests and multivariable linear or median regression models will be used. Multivariable models will adjust for study center, type of chemotherapy, type of cancer, gender, and age. Intention-to-treat analyses will be conducted based on complete cases and on multiply imputed data using a conditional imputation [48].

\section{Discussion}

\section{Expected key results}

To date there is no prevention or effective treatment for neuropathies though it presents a diagnostic dilemma as physicians need to find the balance between patients' quality of life and the effectiveness of medical therapy. Our main study aim is therefore to evaluate the potential of sensorimotor training and whole-body vibration to prevent CIPN. We expect that both interventions (SMT and WBV) will be able to prevent or at least postpone the incidence of CIPN and in case of occurrence at least reduce the severity of subjective and objective CIPNrelated symptoms such as loss of peripheral deep sensitivity, pain, weakened or absent reflexes or loss of balance control, enabling patients to receive their planned medical therapy. A successful implementation would therefore be of high clinical relevance.

\section{Benefits and risks}

Patients have the potential benefit of being able to prevent the incidence of CIPN or at least reduce their debilitating symptoms of CIPN without any further side-effects. We do not expect any complications. The interventions have no negative influence on their medical therapy. All groups receive the best medical standard. However, we have to account for the possibility 
that patients with neuropathic pain in the lower extremities may possibly experience some pain during the vibration exercises at higher frequencies. Due to the low submaximal intensity, the position taken on the platform, and the well-established, non-invasive assessment methods, we believe the possible risk is very low for patients. The electroneurography is a neurological routine assessment that is not associated with any specific risk. Due to the fact that electricity is used, it is possible that some patients may experience this sensation as uncomfortable or painful.

\section{Potential for bias}

In an exercise intervention study, where patients have to be trained and supervised by qualified exercise therapists, patients are aware of their allocation to the treatment or control group. It is therefore essential that investigators performing the assessments are blinded as to which arm patients are in and are not allowed to train the patients and vice versa. All measurements are performed using highly standardized procedures. Assessments are standardized as well as aligned among the investigators. Patients will additionally be asked not to reveal the result of randomisation to any investigator except of course to the exercise therapist. The study can therefore be considered single-blinded. To further reduce bias, all three centres are equipped with identical technology enabling optimal conditions for comparable data collection. The study coordinator (FS) is the same for all study centres and training of study assistants is identical. All assessments within an individual are always performed by the same trained investigator. Assessments are performed according to standardized operating procedures, at the same time of day, in the same room and maintaining a consistent temperature. Regular meetings are held to optimize coordination of data collection and collaboration among the study centres. Follow-up measurements will be carried out by investigators who are unaware of the treatment allocation, resulting in an unbiased assessment of the outcome. A randomized study design will essentially rule out confounding.

\section{Perspectives}

Our results may contribute to improved supportive care in oncology, thereby enhancing quality of life, enabling the optimal medical therapy in neuropathic cancer patients and, eventually, possibly even improving survival for these patients.

We furthermore expect that the proposed interventions will lead to an improvement of motor and sensory functions (such as balance control, coordination, sensitivity, reflexes, pain) impacted by CIPN. It will help understand the underlying mechanisms of SMT and WBV on motor and sensory functions impaired by PNP.
It could assure best clinical outcomes by improving the side-effects of CIPN without interfering with the planned therapy regime, impacting supportive care for cancer patients. Patients' mobility, autonomy and activities of daily living could be maintained. Consequently, patients' quality of life would be increased. Further possible side-effects (e.g., fatigue) could be decreased and secondary diseases reduced. Additionally, patients' social reintegration could be enhanced. The results can help develop recommendations for patients suffering from CIPN, improving supportive care for cancer patients.

We furthermore aim at publishing the results in peerreviewed scientific journals, raising the awareness of the scientific community for this topic. Furthermore, we will create guidelines, training recommendations, and manuals for clinical practice and health care professionals that can directly be translated into patients' everyday lives. Finally, our results will form the foundation for future research on this topic.

\section{Abbreviations \\ CIPN: Chemotherapy-induced peripheral neuropathy; EORTC-QLQ- \\ C30: European Organisation for Research and Treatment of Cancer - Quality of Life Questionnaire - 30 item core questionnaire; EORTC-QLQ- \\ CIPN20: European Organisation for Research and Treatment of Cancer - Quality of Life Questionnaire - 20 item CIPN-specific questionnaire; FACT- GOG-Ntx: Functional Assessment of Cancer Therapy/Gynaecology Oncology Group - Neurotoxity; FFKA: Freiburger Fragebogen für Körperliche Aktivität - level of physical activity questionnaire; PNP: peripheral neuropahy; \\ SMT: Sensorimotor training; WBV: whole-body vibration}

\section{Acknowledgements}

We acknowledge the support of Harald Schubert and Novotec, who are supplying the vibration- and force plates for the duration of the study as well as offering advisory and technical support.

\section{Funding}

This study is funded by the German Cancer Aid (Deutsche Krebshilfe - DKH 70112048), Buschstraße 32, 53,113 Bonn. The study funders have no influence on study design, collection, management, analysis, and interpretation of data, writing of the report, and the decision to submit the report for publication.

\section{Availability of data and materials}

The anonymized datasets used and/or analysed during the current study will be available from the corresponding author on reasonable request.

\section{Authors' contributions}

FS designed the study protocol. WB, FTB, HCL, MH, VR and ML contributed to the design of the study. FS organises the study in all recruiting centres (i.e., recruitment, data collection). FS, TE, TS and $\mathrm{PH}$ are responsible for patient recruitment and contributed to the protocol. $\mathrm{HCL}$ and $\mathrm{MB}$ are responsible for neurological assessments. CK is responsible for the coordination and conduction of the training in Cologne. VR assist FS and WB in all organisational matters. ML is responsible for overall data management and statistical analysis. FS, WB, HCL, $M B$ and $M L$ will furthermore be responsible for data interpretation. FS wrote the present manuscript. All authors revised the study protocol, read and approved the final manuscript. FS is the guarantor.

\section{Ethics approval and consent to participate}

The study has received consent by the Ethics Committee of the German Sport University as well as the University Hospital Cologne (see Table 1 for approvals). Patients are required to give written informed consent prior to any study engagement. 


\section{Consent for publication}

Not applicable

\section{Competing interests}

The authors declare that they have no competing interests.

\section{Publisher's Note}

Springer Nature remains neutral with regard to jurisdictional claims in published maps and institutional affiliations.

\section{Author details}

${ }^{1}$ Institute for Cardiovascular Research and Sports Medicine, German Sport University Cologne, Am Sportpark Müngersdorf 6, 50933 Cologne, Germany. ${ }^{2}$ Department of Sport, Exercise and Health, University of Basel, Birsstr. 320B, 4052 Basel, Switzerland. ${ }^{3}$ Department of Oncology, University Hospital Basel, Petersgraben 4, 4031 Basel, Switzerland. 'Department of Neurology, University Hospital Cologne, Kerpener Straße 62, 50937 Cologne, Germany. ${ }^{5}$ Department I of Internal Medicine, Center of Integrated Oncology Köln Bonn, University Hospital of Cologne, Kerpener Straße 62, 50937 Cologne, Germany. ${ }^{6}$ Department of Epidemiology and Preventive Medicine, University of Regensburg, Franz-Josef-Strauss-Allee 11, 93053 Regensburg, Germany. ${ }^{7}$ Onkologie Köln, Outpatient clinic for Hematology and Oncology, Sachsenring 69, 50677 Cologne, Germany. ${ }^{8}$ Department of Oncology, St.Antonius-Hospital, Dechant-Decker-Str. 8, 52249 Eschweiler, Switzerland.

Received: 27 July 2017 Accepted: 30 November 2017

Published online: 10 January 2018

\section{References}

1. Pietrangeli A, Leandri M, Terzoli E, Jandolo B, Garufi C. Persistence of highdose oxaliplatin-induced neuropathy at long-term follow-up. Eur Neurol. 2006;56(1):13-6.

2. Lehky TJ, Leonard GD, Wilson RH, Grem JL, Floeter MK. Oxaliplatin-induced neurotoxicity: acute hyperexcitability and chronic neuropathy. Muscle Nerve. 2004;29(3):387-92.

3. Richardson JK, Ashton-Miller JA. Peripheral neuropathy: an often-overlooked cause of falls in the elderly. Postgrad Med. 1996;99(6):161-72.

4. Vogt TKJ, Barth J, Ingel K. Klinische Relevanz und Therapie von therapieassotiierten Polyneuropathien bei Patienten mit Tumorerkrankung In: Arbeitsgemeinschaft für Krebserkrankungen; 2010.

5. Stubblefield MD, Burstein HJ, Burton AW, Custodio CM, Deng GE, Ho M, Junck L, Morris GS, Paice JA, Tummala S, et al. NCCN task force report: management of neuropathy in cancer. Journal of the National Comprehensive Cancer Network: JNCCN. 2009;7(Suppl 5):S1-S26. quiz S27-28

6. Quasthoff S, Hartung HP. Chemotherapy-induced peripheral neuropathy. J Neurol. 2002;249(1):9-17.

7. Tofthagen C, Visovsky C, Berry DL. Strength and balance training for adults with peripheral neuropathy and high risk of fall: current evidence and implications for future research. Oncol Nurs Forum. 2012;39(5):E416-24.

8. Uceyler N, Rogausch JP, Toyka KV, Sommer C. Differential expression of cytokines in painful and painless neuropathies. Neurology. 2007;69(1):42-9.

9. Kaley TJ, Deangelis LM. Therapy of chemotherapy-induced peripheral neuropathy. Br J Haematol. 2009;145(1):3-14

10. Tofthagen C, Overcash J, Kip K. Falls in persons with chemotherapy-induced peripheral neuropathy. Support Care Cancer. 2012;20(3):583-9.

11. Smith EM, Cohen JA, Pett MA, Beck SL. The reliability and validity of a modified total neuropathy score-reduced and neuropathic pain severity items when used to measure chemotherapy-induced peripheral neuropathy in patients receiving taxanes and platinums. Cancer Nurs. 2010;33(3):173-83.

12. Smith BH, Torrance $\mathrm{N}$, Bennett MI, Lee AJ. Health and quality of life associated with chronic pain of predominantly neuropathic origin in the community. Clin J Pain. 2007;23(2):143-9.

13. Wonders KY, Reigle BS, Drury DG. Treatment strategies for chemotherapyinduced peripheral neuropathy: potential role of exercise. Oncol Rev. 2010;4: 117-25.

14. Streckmann F, Kneis S, Leifert JA, Baumann FT, Kleber M, Ihorst G, Herich L Grussinger V, Gollhofer A, Bertz H. Exercise program improves therapyrelated side-effects and quality of life in lymphoma patients undergoing therapy. Ann Oncol. 2014;25(2):493-9.
15. Streckmann F, Zopf EM, Lehmann HC, May K, Rizza J, Zimmer P, Gollhofer A, Bloch W, Baumann FT. Exercise intervention studies in patients with peripheral neuropathy: a systematic review. Sports Med. 2014;44(9):1289-304.

16. Taube W, Gruber M, Beck S, Faist M, Gollhofer A, Schubert M. Cortical and spinal adaptations induced by balance training: correlation between stance stability and corticospinal activation. Acta Physiol (Oxf). 2007;189(4):347-58.

17. Gruber M, Gruber SB, Taube W, Schubert M, Beck SC, Gollhofer A. Differential effects of ballistic versus sensorimotor training on rate of force development and neural activation in humans. J Strength Cond Res. 2007; 21(1):274-82.

18. Freeman MA, Dean MR, Hanham IW. The etiology and prevention of functional instability of the foot. J Bone Joint Surg Br. 1965;47(4):678-85.

19. Verhagen E, van der Beek A, Twisk J, Bouter L, Bahr R, van Mechelen W. The effect of a proprioceptive balance board training program for the prevention of ankle sprains: a prospective controlled trial. Am J Sports Med. 2004;32(6):1385-93.

20. Mynark RG, Koceja DM. Down training of the elderly soleus $\mathrm{H}$ reflex with the use of a spinally induced balance perturbation. J Appl Physiol. 2002; 93(1):127-33.

21. Granacher U, Gollhofer A, Strass D. Training induced adaptations in characteristics of postural reflexes in elderly men. Gait Posture. 2006;24(4):459-66.

22. Steimann M, Kerschgens C, Barth J. Rehabilitation bei Chemotherapieinduzierter Polyneuropathie. Onkologe. 2011;17:940-7.

23. Wampler MA, Hamolsky D, Hamel K, Melisko M, Topp KS. Case report: painful peripheral neuropathy following treatment with docetaxel for breast cancer. Clin J Oncol Nurs. 2005;9(2):189-93.

24. Kawanabe K, Kawashima A, Sashimoto I, Takeda T, Sato Y, Iwamoto J. Effect of whole-body vibration exercise and muscle strengthening, balance, and walking exercises on walking ability in the elderly. The Keio journal of medicine. 2007:56(1):28-33.

25. Bogaerts A, Delecluse C, Boonen S, Claessens AL, Milisen K, Verschueren SM. Changes in balance, functional performance and fall risk following whole body vibration training and vitamin D supplementation in institutionalized elderly women. A 6 month randomized controlled trial. Gait Posture. 2011;33(3):466-72.

26. Rittweger J. Vibration as an exercise modality: how it may work, and what its potential might be. Eur J Appl Physiol. 2010;108(5):877-904.

27. Kirchner E. Pflegerische Interventionen und Möglichkeiten bei krebstherapiebedingter Polyneuropathie. DLH-INFO. 2008:37:19-21.

28. Blottner D, Salanova M, Puttmann B, Schiffl G, Felsenberg D, Buehring B, Rittweger J. Human skeletal muscle structure and function preserved by vibration muscle exercise following 55 days of bed rest. Eur J Appl Physiol. 2006;97(3):261-71.

29. Lau RW, Liao LR, Yu F, Teo T, Chung RC, Pang MY. The effects of whole body vibration therapy on bone mineral density and leg muscle strength in older adults: a systematic review and meta-analysis. Clin Rehabil. 2011;25(11):975-88.

30. Cochrane DJ. Vibration exercise: the potential benefits. Int J Sports Med. 2011:32(2):75-99.

31. Spiliopoulou SI, Amiridis IG, Tsigganos G, Economides D, Kellis E. Vibration effects on static balance and strength. Int J Sports Med. 2010;31(9):610-6.

32. Schonsteiner SS, Bauder Missbach H, Benner A, Mack S, Hamel T, Orth M, Landwehrmeyer B, Sussmuth SD, Geitner C, Mayer-Steinacker R, et al. A randomized exploratory phase 2 study in patients with chemotherapyrelated peripheral neuropathy evaluating whole-body vibration training as adjunct to an integrated program including massage, passive mobilization and physical exercises. Exp Hematol Oncol. 2017;6:5.

33. Anna $\mathrm{H}$. The effects of sensorimotor-training and whole body vibration on patients with chemotherapy induced peripheral neuropathy in the aftercare- a pilot study. Cologne: University of Cologne; 2014.

34. Pataky Z, Herrmann FR, Regat D, Vuagnat $H$. The at-risk foot concerns not only patients with diabetes mellitus. Gerontology. 2008;54(6):349-53.

35. White CM, Pritchard J, Turner-Stokes L. Exercise for people with peripheral neuropathy. Cochrane Database Syst Rev. 2004;(4):CD003904. https://doi. org/10.1002/14651858.CD003904.pub2.

36. Coren S. The lateral preference inventory for measurement of handedness, footedness, eyedness, and earedness: norms for young adults. Bull Psychon Soc. 1993;31:1-3.

37. Taube W, Gruber M, Gollhofer A. Spinal and supraspinal adaptations associated with balance training and their functional relevance. Acta Physiol (Oxf). 2008;193(2):101-16.

38. Hayes S, Newman B. Exercise in cancer recovery: An overview of the evidence. Cancer Forum. 2006;30:13-7. 
39. Aaronson NKAS, Bergman B, Bullinger M, Cull A, Duez NJ, Filiberti A, Flechtner H, Fleishman SBHJ, Kaasa S, Klee MC, Osoba D, Razavi D, Rofe PB, Schraub S, SM SKCA, Takeda F. The European Organisation for Research and Treatment of Cancer QLQ-C30: A quality-of-life instrument for use in international clinical trials in oncology. J Natl Cancer Inst. 1993;85:365-76.

40. Emery CF. Cognitive functioning among patients in cardiopulmonary rehabilitation. J Cardpulm Rehabil. 1997;17(6):407-10.

41. Freynhagen R, Baron R, Gockel U, Tolle TR. painDETECT: a new screening questionnaire to identify neuropathic components in patients with back pain. Curr Med Res Opin. 2006;22(10):1911-20.

42. Bennett Ml, Attal N, Backonja MM, Baron R, Bouhassira D, Freynhagen R, Scholz J, Tolle TR, Wittchen HU, Jensen TS. Using screening tools to identify neuropathic pain. Pain. 2007;127(3):199-203.

43. Frey IBA, Grathwohl D, Keul J. Freiburger questionnaire of physical activitydevlopment, evaluation and application. SozPraventivmed. 1999:44(2):55-64

44. Ainsworth BE, Haskell WL, Herrmann SD, Meckes N, Bassett DR Jr, TudorLocke C, Greer JL, Vezina J, Whitt-Glover MC, Leon AS. 2011 compendium of physical activities: a second update of codes and MET values. Med Sci Sports Exerc. 2011;43(8):1575-81.

45. Pocock SJ, Simon R. Sequential treatment assignment with balancing for prognostic factors in the controlled clinical trial. Biometrics. 1975;31(1):103-15.

46. Barthel FM, Babiker A, Royston P, Parmar MK. Evaluation of sample size and power for multi-arm survival trials allowing for non-uniform accrual, nonproportional hazards, loss to follow-up and cross-over. Stat Med. 2006; 25(15):2521-42.

47. Royston P, Barthel FM-S. Projection of power and events in clinical trials with a time-to-event outcome. Stata J. 2010;10(3):386.

48. White IR, Carpenter J, Horton NJ. Including all individuals is not enough: lessons for intention-to-treat analysis. Clin Trials. 2012;9(4):396-407.

\section{Submit your next manuscript to BioMed Central and we will help you at every step:}

- We accept pre-submission inquiries

- Our selector tool helps you to find the most relevant journal

- We provide round the clock customer support

- Convenient online submission

- Thorough peer review

- Inclusion in PubMed and all major indexing services

- Maximum visibility for your research

Submit your manuscript at www.biomedcentral.com/submit

C) Biomed Central 